\title{
TIGRIDIA RZEDOWSKIANA (TIGRIDIEAE: IRIDACEAE), UNA NUEVA ESPECIE DEL ESTADO DE QUERÉTARO, MÉXICO
}

\author{
Aarón Rodríguez y Luis Ortiz-Catedral \\ Departamento de Botánica y Zoología, Universidad de Guadalajara \\ Apartado postal 139, 45101 Zapopan, Jalisco, México
}

\section{RESUMEN}

Se describe a Tigridia rzedowskiana (Tigridieae: Iridaceae) como una especie nueva para la ciencia procedente del estado de Querétaro, México. Se caracteriza por sus flores amarillentas con máculas purpúreas, los tépalos externos obovados, obtusos a apiculados y los tépalos internos deltado-hastados y apiculados. Sus flores abren durante la mañana. Tigridia rzedowskiana es morfológicamente similar a $T$. catarinensis y se discuten sus diferencias.

Palabras clave: Iridaceae, México, Tigridia.

\section{ABSTRACT}

Tigridia rzedowskiana (Tigridieae: Iridaceae) is a new species found in the state of Querétaro, Mexico. It is unique by its yellowish flowers with maroon spots, outer tepals obovate, obtuse to apiculate and inner tepals deltate-hastate and apiculate. The flowers open early in the morning. Tigridia rzedowskiana is morphologically similar to T. catarinensis and we discuss their differences.

Key words: Iridaceae, Mexico, Tigridia.

El conocimiento de las especies del género Tigridia Juss. (Tigridieae: Iridaceae) y su distribución en México es incompleto. Durante la revisión de material de herbario es común encontrar ejemplares procedentes de localidades no registradas con anterioridad para los taxa revisados (Rodríguez et al., 1996; Rodríguez y OrtizCatedral, 2002; Rodríguez et al., 2003). También es frecuente notar que la preservación de las estructuras florales con importancia taxonómica es deficiente en los especímenes de herbario, lo que hace necesaria la observación detallada de material fresco para confirmar la identidad taxonómica de los mismos. En ocasiones, 
lo anterior conduce a encontrar especies nuevas para la ciencia. Rodríguez y OrtizCatedral (2002) informaron sobre la presencia en el estado de Querétaro de $T$. catarinensis Cruden, especie conocida sólo del estado de San Luis Potosí (Cruden, 1975; Espejo-Serna y López-Ferrari, 1996a; 1996b; Rodríguez y Ortiz-Catedral, 2002). En el mes de julio del 2003, se recolectó material fresco procedente de la localidad de Querétaro citada por Rodríguez y Ortiz-Catedral (2002) y se realizó una comparación con el procedente de la localidad tipo en San Luis Potosí. Nuestra conclusión es que las plantas encontradas en Querétaro y previamente determinadas como T. catarinensis pertenecen a una especie nueva para la ciencia y cuya descripción es la siguiente:

Tigridia rzedowskiana Aarón Rodr. \& L. Ortiz-Catedral, sp. nov. (Fig. 1; Figs. 2A, 2B)

Herba perennis, bulbosa, erecta; rami 1-3, in rhipidio terminati; caules $40-70 \mathrm{~cm}$ alti; bulbus ovoideus $5-6 \mathrm{~cm}$ longus et $2.5-3 \mathrm{~cm}$ latus, tunicis brunneis; folia basalia bina, $27-60 \mathrm{~cm}$ longa, $0.6-3 \mathrm{~cm}$ lata; folium caulis lineare, $22-33 \mathrm{~cm}$ longum, 0.8-2.2 cm latum; riphidia 1-3; flores plures; tepala conniventia ad basim cupulam formantia, cupula luteola, cum maculis marroninis; tepala externa obovata, ad basim concava, cuneata, apiculata, luteola, cum maculis marroninis, supra cupulam patente atromarronina, $2.5 \mathrm{~cm}$ longa et $1.5 \mathrm{~cm}$ lata; tepala interna deltato-hastata, concava, unguiculata, ungue $4 \mathrm{~mm}$ longo, apiculata, luteola, cum maculis marroninis, 2-2.2 cm longa et $1.3 \mathrm{~cm}$ lata; glandula ad partem basalem tepalorum posita, concolor; filamenta connata, $9-11 \mathrm{~mm}$ longa; antherae liberae oblongae, procurrentes, 9-10 mm longae; styli ramuli 5-7 mm longi, bipartiti, luteoli, sinubus styli ramis mucrone 2.7-3 mm longo instructis; ovarium $8 \mathrm{~mm}$ longum; capsula oblonga, clavata, $3 \mathrm{~cm}$ longa et $10 \mathrm{~mm}$ lata; semina ignota.

Hierba perenne, bulbosa, erecta; ramas 1-3, cada una terminada en un ripidio; escapo floral de 40-70 cm de largo; bulbos ovoides, de 5-6 cm largo, 2.5-3 cm ancho; túnicas de color pardo oscuro; hojas basales dos, de 27-60 cm largo, 0.6-3 cm ancho; hoja caulinar única, de $22-33 \mathrm{~cm}$ largo, 0.8-2.2 cm ancho; inflorescencia consistente de 1-3 ripidios de 4.5-6.6 $\mathrm{cm}$ de largo; los ripidios secundarios emergen de la base de la hoja caulinar; brácteas del ripidio subiguales, de 4.5-6.6 cm largo, 1.2-1.3 cm ancho; pedicelos de 2-6 cm largo; flores erectas, tépalos conniventes en la base formando una taza somera; tépalos externos de $2.5 \mathrm{~cm}$ de largo, $1.5 \mathrm{~cm}$ de ancho, obovados, cóncavos, cuneados, obtusos a apiculados, amarillentos con máculas purpúreas, la parte distal refleja, uniformemente coloreada de púrpura, salvo algunas máculas amarillentas; tépalos internos de $2-2.2 \mathrm{~cm}$ de largo, $1.3 \mathrm{~cm}$ de ancho, deltado-hastados, cóncavos, unguiculados, uña de $4 \mathrm{~mm}$ de largo, apiculados, con 
Rodríguez y Ortiz-Catedral: Tigridia rzedowskiana una nueva especie de Querétaro

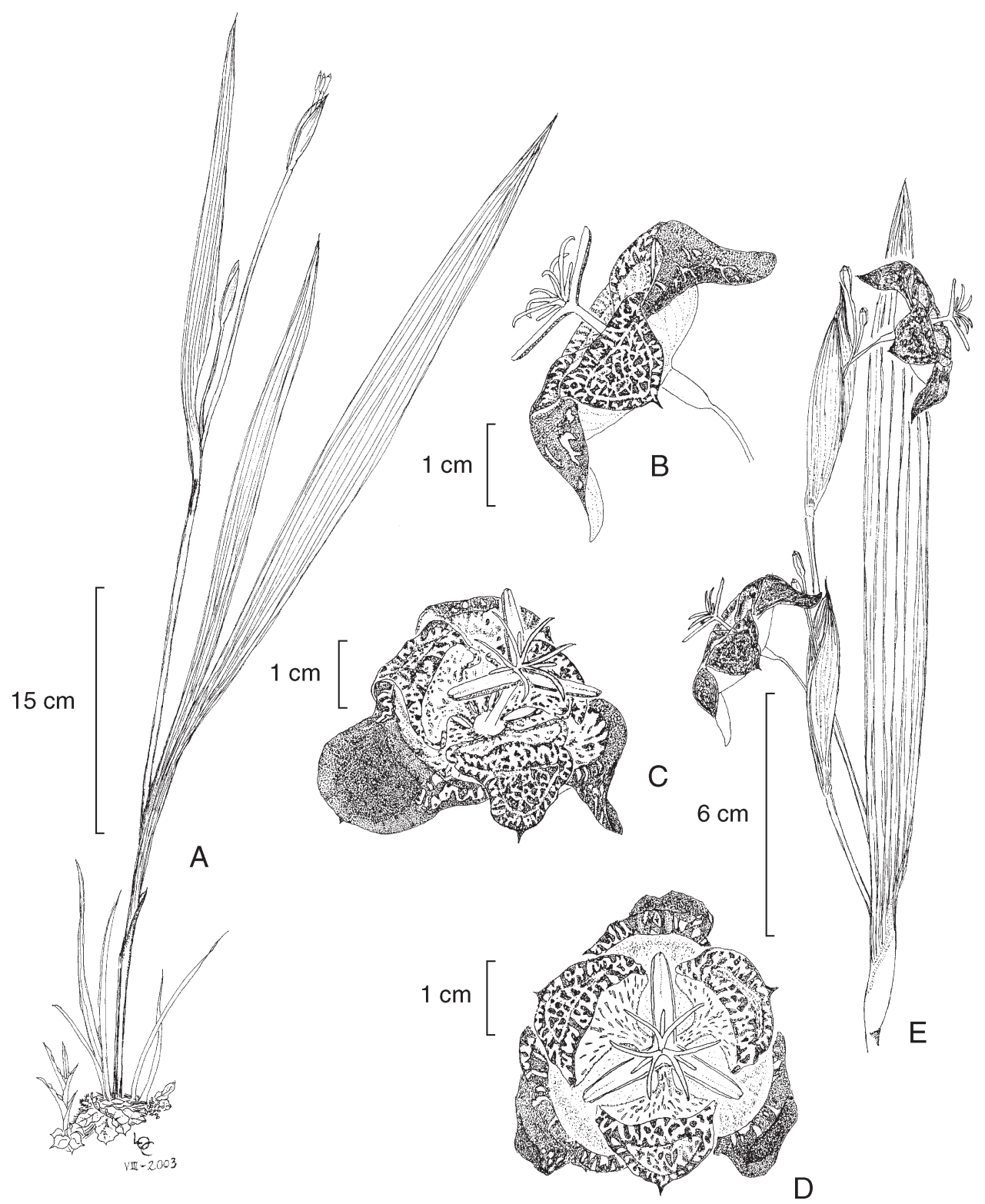

Fig. 1. Tigridia rzedowskiana. A. Hábito; B. Flor, vista lateral; C. Flor, vista semifrontal; D. Flor, vista frontal; E. Detalle de la hoja caulinar y los ripidios. A-E basados en el holótipo. Ilustración elaborada por L. Ortiz-Catedral. 


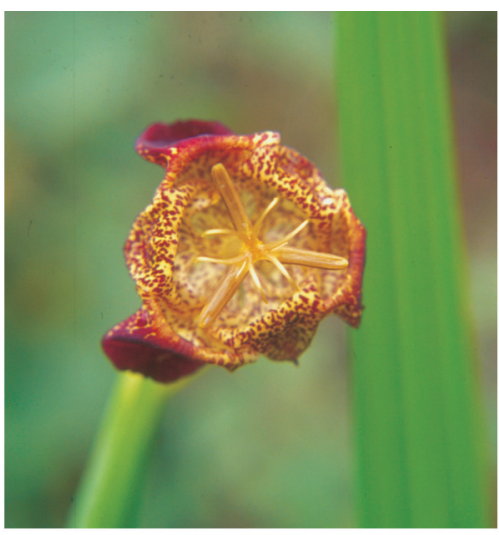

A

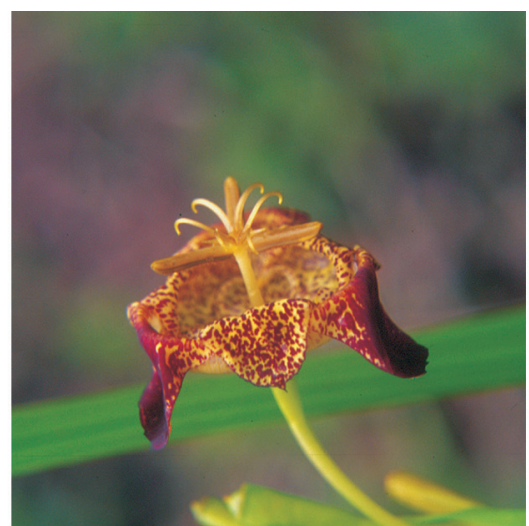

B

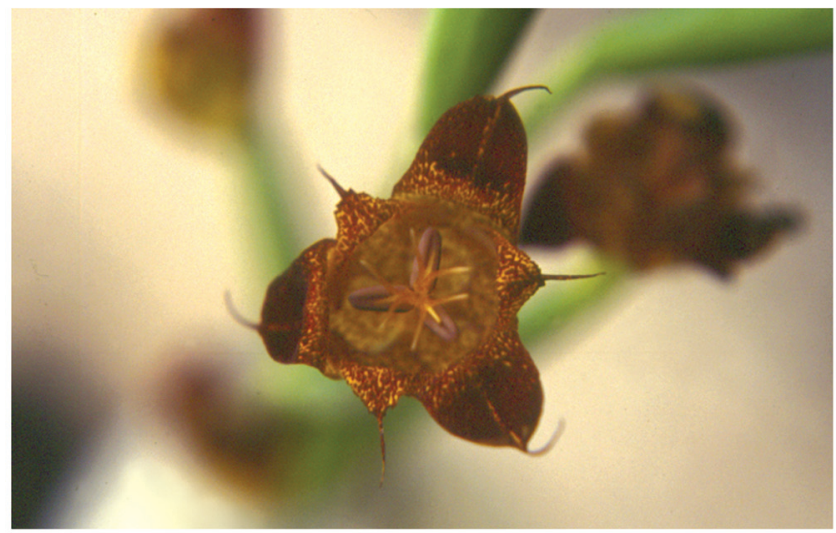

C

Fig. 2. Tigridia rzedowskiana (A, B) y T. catarinensis (C). A y B son fotografías del holótipo tomadas por A. Rodríguez. C es una fotografía tomada en la localidad tipo por A. Rodríguez (A. Rodríguez 2718, IBUG).

máculas purpúreas sobre fondo amarillento; nectarios concoloros, situados en la base de los tépalos internos; columna estaminal de 9-11 mm de largo; anteras oblongas, perpendiculares a la columna estaminal, de 9-10 mm largo; ramas del estilo de 5-7 mm de largo, bífidas, amarillentas, con un mucrón de 2.7-3 $\mathrm{mm}$ de largo inserto en la base de la bifurcación; ovario de $8 \mathrm{~mm}$ de largo; fruto oblongo, claviforme, de $3 \mathrm{~cm}$ de largo, $10 \mathrm{~mm}$ de ancho; semillas no vistas.

Tipo: México: Querétaro, municipio de Pinal de Amoles, El Cajón o Puerto del Pino, $3 \mathrm{~km}$ al SW de Pinal de Amoles, frente a la brecha que sube a la estación 
de microondas, 2420 m s.n.m., bosque perturbado de Pinus patula, Cupressus y Quercus, 17.VII.2003. A. Rodríguez y L. Ortiz-Catedral 3134 (holótipo: IBUG; isótipos: IEB, MEXU, UAMIZ).

Material adicional examinado: México: Querétaro, municipio de Pinal de Amoles, Puerto del Pino, Pinal de Amoles, $2560 \mathrm{~m}$, bosque de Pinus patula, reforestado y muy perturbado, 7.VII.1994. S. Zamudio y E. PérezCalix 9306 (IEB).

Distribución y hábitat: Tigridia rzedowskiana se conoce únicamente de la localidad tipo. Crece en laderas rocosas soleadas o en semisombra en bosques de Quercus y Pinus entre los 2420 y los 2560 m s.n.m. Otros taxones asociados con la especie son Echeandia, Echeveria, Physalis y Solanum. El suelo es arcilloso con una capa gruesa de materia orgánica. En los alrededores se practica el cultivo de maguey y el pastoreo.

Etimología. El epíteto específico hace referencia al Dr. Jerzy Rzedowski Rotter, el botánico mexicano más destacado.

Tigridia rzedowskiana es morfológicamente similar a $T$. catarinensis. El tamaño de las estructuras florales y otras características de ambas especies se presentan en en el cuadro 1 y la figura 2. Las dos especies son semejantes en el tamaño de las plantas y el color de las flores. Sin embargo, T. rzedowskiana tiene sólo una hoja caulinar, mientras T. catarinensis presenta dos. En T. rzedowskiana, los tépalos externos son más anchos y los tépalos internos más largos y anchos que en $T$. catarinensis. Además, T. rzedowskiana presenta limbos bien desarrollados en los tépalos internos mientras que en $T$. catarinensis estos están reducidos. Asimismo, en T. rzedowskiana los tépalos externos son obovados, obtusos o apiculados y los internos son hastados y apiculados. En contraste, en T. catarinensis, los tépalos externos son oblongos y cirrosos y los internos hastados y cirrosos. También existen diferencias en el tamaño y posición de las anteras, ya que en $T$. rzedowskiana son más grandes y están extendidas pero en $T$. catarinensis son ascendentes. La columna estaminal y los frutos son más largos en T. rzedowskiana que en $T$. catarinensis. Otra diferencia entre ambas especies consiste en que los nectarios en $T$. catarinensis son blancos, mientras que los de T. rzedowskiana son concoloros respecto a los tépalos internos. Por último, las flores de T. rzedowskiana abren por la mañana y las de $T$. catarinensis lo hacen por la tarde. Únicamente $T$. catarinensis y $T$. dugesii $\mathrm{S}$. Watson comparten esta última característica. Por otro lado, a diferencia de lo acostumbrado por T. rzedowskiana, T. catarinensis habita en lugares más secos, en laderas rocosas soleadas, en bosques de Quercus, 
Cuadro 1. Comparación morfológica entre Tigridia rzedowskiana y T. catarinensis.

\begin{tabular}{|l|c|c|}
\hline \multicolumn{1}{|c|}{ Característica } & T. rzedowskiana & T. catarinensis \\
\hline Hojas basales & 2 & 2 \\
\hline Hojas caulinares & 1 & 2 \\
\hline Antesis & en la mañana & en la tarde \\
\hline $\begin{array}{l}\text { Longitud de la hoja } \\
\text { caulinar superior }\end{array}$ & $22-23 \mathrm{~cm}$ & $75 \mathrm{~cm}$ \\
\hline $\begin{array}{l}\text { Longitud del tépalo } \\
\text { externo }\end{array}$ & $25 \mathrm{~mm}$ & $22 \mathrm{~mm}$ \\
\hline Ancho del tépalo externo & $15 \mathrm{~mm}$ & oblongo \\
\hline Forma del tépalo externo & obovado & cirroso \\
\hline Ápice del tépalo externo & obtuso a apiculado & $15 \mathrm{~mm}$ \\
\hline $\begin{array}{l}\text { Longitud del tépalo } \\
\text { interno }\end{array}$ & $20-22 \mathrm{~mm}$ & $7-9 \mathrm{~mm}$ \\
\hline Ancho del tépalo interno & $13 \mathrm{~mm}$ & hastado \\
\hline Forma del tépalo interno & hastado & cirroso \\
\hline Ápice del tépalo interno & apiculado & blanco \\
\hline Color del nectario & sin color & $7 \mathrm{~mm}$ \\
\hline $\begin{array}{l}\text { Longitud de la columna } \\
\text { estaminal }\end{array}$ & $9-11 \mathrm{~mm}$ & $5 \mathrm{~mm}$ \\
\hline Longitud de las anteras & $9-10 \mathrm{~mm}$ & $1.2 \mathrm{~cm}$ \\
\hline Longitud del fruto & $3 \mathrm{~cm}$ & 8.9 \\
\hline Ancho del fruto & $10 \mathrm{~mm}$ & \\
\hline
\end{tabular}

entre los 1650 y los 1750 m s.n.m., asociada con Agave, Bauhinia, Cuphea, Echeandia y con varias cactáceas.

No obstante la minuciosa revisión de Tigridia para México y Guatemala realizada por Molseed (1970) y un análisis filogenético posterior hecho por Rodríguez (1999), las relaciones de parentesco entre las especies no son claras. Molseed (1970) propuso la división del género en varios complejos de especies con base en caracteres morfológicos. Cruden (1975) describió tres nuevas especies de Tigridia y propuso una reorganización de los complejos multiflora y vanhouttei. De acuerdo con este último esquema, T. rzedowskiana forma parte del complejo multiflora, el cual agrupa especies con las siguientes características: flores erectas o péndulas, crateriformes, con los segmentos del perianto reflejos y ligera a 
marcadamente desiguales, tépalos internos unguiculados y hastados, limbo de los tépalos internos bien formado o reducido; nectarios semicirculares cercanos al borde de la región crateriforme, anteras y ramas del estilo extendidas o ascendentes y frutos subglobosos o claviformes. Las especies incluidas en el complejo multiflora son hasta el momento: T. catarinensis, T. huajuapanensis Molseed ex Cruden, T. illecebrosa Cruden, T. matudae Molseed, T. multiflora (Baker) Ravenna, T. potosina López-Ferrari et Espejo, T. pulchella Rob., T. purpusii Molseed y T. rzedowskiana.

\section{AGRADECIMIENTOS}

Agradecemos al curador del herbario IEB las facilidades otorgadas durante la revisión del material herborizado y depositado en sus colecciones y a los revisores anónimos que han hecho sugerencias al manuscrito.

\section{LITERATURA CITADA}

Cruden, R. W. 1975. New Tigridieae (Iridaceae) from Mexico. Brittonia 27:103-109.

Espejo-Serna, A. y A. R. López-Ferrari. 1996a. Las monocotiledóneas mexicanas, una sinopsis florística. 1. Lista de referencia. Parte VI. Consejo Nacional de la Flora de México, A. C., Universidad Autónoma Metropolitana Iztapalapa, Comisión Nacional para el Conocimiento y Uso de la Biodiversidad. México, D.F. 116 pp.

Espejo-Serna, A. y A. R. López-Ferrari. 1996b. Comentarios florístico-ecológicos sobre las iridáceas mexicanas. Acta Bot. Mex. 34: 25-47.

Molseed, E. 1970. The genus Tigridia (Iridaceae) of Mexico and Central America. Univ. California Pub. Bot. 54: 1-113.

Rodríguez, A. 1999. Molecular and morphological systematics of the 'tiger-flower' group (Tribe Tigridieae: Iridaceae): biogeography and evidence for the adaptative radiation of the subtribe Tigridiinae. Tesis de doctorado, University of Wisconsin-Madison. $225 \mathrm{pp}$.

Rodríguez, A. y L. Ortiz-Catedral. 2002. Nuevos registros de iridáceas mexicanas. Bol. Inform. Inst. Bot. Univ. Guadalajara 9(1-2): 25-36.

Rodríguez, A., L. Ortiz-Catedral y E. Heaton. 2003. Tres nuevas localidades de tigridias endémicas de México: Tigridia bicolor, T. matudae, T. vanhouttei ssp. roldanii. Act. Bot. Mex. 62: 1-8.

Rodríguez, A., O. Vargas, E. Villegas y K. J. Sytsma. 1996. Nuevos informes de iridáceas (Tigridieae) en Jalisco. Bol. Inst. Bot. Univ. Guadalajara 4: 39-47.

Recibido en diciembre de 2003.

Aceptado en marzo de 2005. 